\title{
A GROUP-THEORETIC PROPERTY OF THE EUCLIDEAN METRIC
}

\author{
ROBERT WILLIAMSON AND LUDVIK JANOS
}

\begin{abstract}
Let $d$ denote a metric on $\mathbf{R}^{n}(n \in \mathbf{N})$ compatible with its Euclidean toplogy, and let $I(d)$ be the group of isometries on $\mathbf{R}^{n}$ relative to this metric. We show that whenever $I(d)$ includes the group of motions then $I(d)$ is identical with it.
\end{abstract}

1. Introduction. If $X$ is a metrizable topological space we let $M(X)$ stand for the set of all metrics on $X$ compatible with the topology of $X$ and let $H(X)$ denote the group of all homeomorphisms $T: X \rightarrow X$ of $X$ onto itself. Given $d \in M(X)$ let $I(d) \subset H(X)$ denote the group of isometries relative to $d$. A metric $d \in M(X)$ is said to be maximal if there is no $d^{\prime} \in M(X)$ with $I\left(d^{\prime}\right)$ properly containing $I(d)$. The main purpose of this note is to show that the Euclidean metric on $\mathbf{R}^{n}$ is maximal in this sense. (See Remark 1.)

THEOREM. For every $n \in \mathbf{N}$ the Euclidean metric on $\mathbf{R}^{n}$ is maximal.

2. Proof of the theorem. Using elementary geometric arguments concerning homeomorphisms on $\mathbf{R}$ the proof has been obtained in [3, Theorem 3.4] for $n=1$. Since for $n=2$ these arguments are not applicable it took considerable time to find a hold to draw on. The following result proved by Beckman and Quarles [1] is useful.

TheOREM 2.1. Let $T: \mathbf{R}^{n} \rightarrow \mathbf{R}^{n}, n \geqslant 2$, be a (not necessarily continuous) function from $\mathbf{R}^{n}$ into itself and assume there exists $a>0$ so that, for every $x, y \in \mathbf{R}^{n}$, $\|x-y\|=a$ implies $\|T x-T y\|=a$. Then $T$ is a Euclidean motion, i.e., an isometry on $\mathbf{R}^{n}$ relative to the metric $\|x-y\|$.

Using this powerful statement we prove our theorem as follows. The idea of the proof is due to the first author. We first note that if $d \in M\left(\mathbf{R}^{n}\right)$ is such that $I(d)$ contains the group of motions then $d(x, y)$ must be of the form

$$
d(x, y)=\varphi(\|x-y\|)
$$

where $\varphi$ is a continuous function taking $[0, \infty)$ into itself. This follows from the fact that the Euclidean group is transitive on pairs of equidistant points. The continuity of $\varphi$ follows from the continuity of $d$. If we could assume that $\varphi$ is such that there exists at least one value $b>0$ so that the equation

$$
\varphi(t)=b
$$

Received by the editors June 3, 1985 and, in revised form, September 9, 1985

1980 Mathematics Subject Classification (1985 Revision). Primary 54E35, 54E40, 54H15.

$$
\text { (C1986 American Mathematical Society }
$$
$0002-9939 / 86 \$ 1.00+\$ .25$ per page 
has precisely one solution, then we are done, choosing in Theorem 2.1 for the value $a$ the unique solution of (2). Unfortunately there are metrics for which no such $b$ exists (see Remark 2), so we reason as follows: we claim that there exists at least one value $b>0$ with $\varphi^{-1}(b)$ containing no interval. It is evident that there exists at most a countable set of values $b_{0}, b_{1}, \ldots$, for which $\varphi^{-1}\left(b_{i}\right)$ contains an interval. So choosing the value as above we see that the set $\varphi^{-1}(b)$ is totally disconnected. Assume now that $T \in I(d)$, and let $x \in \mathbf{R}^{n}$ be fixed. Let $S(x, a)$ be the sphere about $x$ with the radius $a \in \varphi^{-1}(b)$,

$$
S(x, a)=\left\{y \in \mathbf{R}^{n}:\|y-x\|=a\right\}
$$

(where we choose the value $a$ arbitrarily). We now consider the mapping $B_{x}$ : $S(x, a) \rightarrow[0, \infty)$ defined by $B_{x}(y)=\|T y-T x\|$ for $y \in S(x, a)$. Since $S(x, a)$ is connected, $T$ continuous and since $\varphi(\|T y-T x\|)=b$ for all $y \in S(x, a)$, we see that the value $B_{x}(y) \in \varphi^{-1}(b)$ must be a constant independent of $y$, say $a^{*}(x)$. Now we prove that $a^{*}(x)$ is also independent of $x$, i.e., $B_{x}(y)$ is a universal constant. If $\left\|x_{1}-x\right\|<a$ then the bisector set $\left\{s:\|x-s\|=\left\|s-x_{1}\right\|\right\}$ meets $S(x, a)$ in a point, say $w \in S(x, a)$, and we have that $x, x_{1} \in S(w, a)$. Consequently, $B_{w}(x)=B_{w}\left(x_{1}\right)$ $=a^{*}(w)$ by the result above. But since $w \in S(x, a)$ and $w \in S\left(x_{1}, a\right)$, we have that $a^{*}(w)=a^{*}(x)=a^{*}\left(x_{1}\right)$ which shows that $a^{*}(x)$ is locally constant. Since $\mathbf{R}^{n}$ is connected, $a^{*}$ is a constant and we arrived at the relation:

$$
\|x-y\|=a \text { implies }\|T x-T y\|=a^{*},
$$

from which follows that $\left(a / a^{*}\right) T$ is a motion and therefore belongs to $I(d)$. Since $T^{-1} \in I(d)$ this implies that the similarity $S$ defined by $x \rightarrow\left(a / a^{*}\right) x$ also belongs to $I(d)$, and we shall show that $a^{*} \neq a$ leads to contradiction. We consider the one-dimensional subspace $\mathbf{R} \subseteq \mathbf{R}^{n}$ with $d_{1} \in M(\mathbf{R})$ defined as $d$ restricted to $\mathbf{R}$. Since the similarity $S$ leaves $\mathbf{R}$ invariant we can apply Theorem 3.4 [3] to show that $a^{*} \neq a$ is impossible, implying that $T$ is a motion of $\mathbf{R}^{n}$, which completes our proof.

REMARK 1. To show there are metrics on $\mathbf{R}^{n}$ which are not maximal we exhibit metrics which possess just the opposite property; they do not have any symmetry at all since the only isometry they admit is the identity. We shall call such metrics rigid and offer the following method of constructing them: We choose a topological embedding $i: \mathbf{R}^{n} \rightarrow \mathbf{R}^{n+1}$ such that the image $i\left(\mathbf{R}^{n}\right)$ does not lie in any affine subspace of dimension $<n+1$ and such that $i\left(\mathbf{R}^{n}\right)$ is not invariant by any motion in $\mathbf{R}^{n+1}$ except the identity. Then the metric $d$ on $\mathbf{R}^{n}$ defined by $d(x, y)=\| i(x)-$ $i(y) \|$ is rigid. This follows from Lemma 1 of [4] (see also [3, §38]). As a simple example we may construct a rigid metric on $\mathbf{R}$ by mapping it on the letter " $L$ " with deleted end points situated in the plane.

REMARK 2. There exist continuous maps $\varphi[0, \infty) \rightarrow[0, \infty)$ such that $\varphi^{-1}(y)$ has cardinality $\neq 1$ for any $y \neq 0$, but for which $\varphi \circ d$ is a metric equivalent to $d$, where $d$ is the Euclidean metric. This fact is perhaps not quite trivial, so we provide a construction. In order for $\varphi \circ d$ to be an equivalent metric it is sufficient that $\varphi^{-1}(0)=\{0\}$ and that $\varphi$ have the "triangle property": if $x, y, z \in[0, \infty)$, and if $x+y \geqslant z, x+z \geqslant y$, and $z+y \geqslant x$, then $\varphi(x)+\varphi(y) \geqslant \varphi(z), \varphi(x)+\varphi(z) \geqslant$ $\varphi(y)$, and $\varphi(z)+\varphi(y) \geqslant \varphi(x)$. Now one can show that $\varphi$ will have this property if 
it satisfies the following "decreasing slope"condition: For any $x, y, z \in[0, \infty)$, if $x \leqslant z$, then

$$
\varphi(x) / x \geqslant(\varphi(y)-\varphi(z)) /(y-z)
$$

and if $x \leqslant \frac{1}{2} z$, in addition,

$$
\varphi(x) / x \geqslant-(\varphi(y)-\varphi(z)) /(y-z) .
$$

One can now construct $\varphi$ inductively. Let $\varphi_{n}$ be a sequence with $\varphi_{1}=1$, monotonically strictly decreasing to 0 , such as $\varphi_{n} \leqslant 1 / n$. We will define by induction another sequence of the same sort, $t_{n}$, and we define $\varphi$ so that $\varphi\left(t_{2 k+1}\right)=\varphi_{k}$, $\varphi\left(t_{2 k}\right)=\varphi_{k+1}$, and on each segment $\left[t_{j+1}, t_{j}\right], \varphi$ is linear; so it alternately increases and decreases. So for $t \geqslant 1$, set $\varphi(t)=1$, set $t_{1}=1$, and if $t q$ is defined for $q \leqslant j$, define $t_{j+1}$ by

(i) if $j=2 k+1$, then $t_{j+1}=\varphi_{k+2} / \varphi_{k+1}$ and $\varphi(t)=t \varphi_{k+1}$, on $\left[t_{j+1}, t_{j}\right]$;

(ii) if $j=2 k$, then $t_{j+1}$ is the unique point such that the slope of the line through the origin to $\left(t_{j+1}, \varphi_{k}\right)$ is the negative of the slope of the line from $\left(t_{2 k+1}, \varphi_{k}\right)$ to $\left(t_{2 k}, \varphi_{k+1}\right)$, and on $\left[t_{j+1}, t_{j}\right], \varphi$ is the latter segment.

\section{REFERENCES}

1. T. S. Beckman and D. A. Quarles, Jr., On isometries of Euclidean spaces, Proc. Amer. Math. Soc. 4 (1953), 810-815.

2. L. M. Blumenthal, Theory and applications of distance geometry, Oxford University Press, 1953.

3. Ludvik Janos, On maximal groups of isometries, Proc. Amer. Math. Soc. 28 (1971), 584-586.

4. Daryl Tingley, Metric transformations on the real line, Rocky Mountain J. Math 15 (1985), 199-206.

Department of Mathematics, Claremont Graduate School, Claremont, California 91711

Department of Mathematics, California State University, Long Beach, California 90804 